\title{
Sex differences in the human peripheral blood transcriptome
}

\author{
Rick Jansen ${ }^{1 *}$, Sandra Batista ${ }^{5}$, Andrew I Brooks ${ }^{4}$, Jay A Tischfield ${ }^{4}$, Gonneke Willemsen ${ }^{2}$, Gerard van Grootheest ${ }^{1}$, \\ Jouke-Jan Hottenga ${ }^{2}$, Yuri Milaneschi ${ }^{1}$, Hamdi Mbarek ${ }^{2}$, Vered Madar ${ }^{3}$, Wouter Peyrot ${ }^{1}$, Jacqueline M Vink ${ }^{2}$, \\ Cor L Verweij ${ }^{6}$, Eco JC de Geus ${ }^{2}$, Johannes H Smit ${ }^{1}$, Fred A Wright ${ }^{3}$, Patrick F Sullivan ${ }^{5}$, Dorret I Boomsma ${ }^{2 \dagger}$ \\ and Brenda WJH Penninx ${ }^{1+}$
}

\begin{abstract}
Background: Genomes of men and women differ in only a limited number of genes located on the sex chromosomes, whereas the transcriptome is far more sex-specific. Identification of sex-biased gene expression will contribute to understanding the molecular basis of sex-differences in complex traits and common diseases.

Results: Sex differences in the human peripheral blood transcriptome were characterized using microarrays in 5,241 subjects, accounting for menopause status and hormonal contraceptive use. Sex-specific expression was observed for 582 autosomal genes, of which $57.7 \%$ was upregulated in women (female-biased genes). Female-biased genes were enriched for several immune system GO categories, genes linked to rheumatoid arthritis (16\%) and genes regulated by estrogen (18\%). Male-biased genes were enriched for genes linked to renal cancer (9\%). Sex-differences in gene expression were smaller in postmenopausal women, larger in women using hormonal contraceptives and not caused by sex-specific eQTLs, confirming the role of estrogen in regulating sex-biased genes.
\end{abstract}

Conclusions: This study indicates that sex-bias in gene expression is extensive and may underlie sex-differences in the prevalence of common diseases.

\section{Background}

Sexual dimorphism extends into marked cellular, metabolic, physiological and anatomical differences and leads to sex differences in disease prevalence, expression and severity of, for example, cardiovascular [1], and autoimmune [2] diseases, personality [3] and psychiatric disorders [4]. Sex inequalities are an increasingly recognized challenge in both basic research and clinical medicine [5], and understanding the molecular mechanisms behind sex differences may lead to new insights into sex-specific pathophysiology and treatment opportunities [6].

Sex differences at the DNA sequence level are restricted to the sex chromosomes. On the X-chromosome, most genes are equally expressed across sex due to X-inactivation in women [7]. The few unshared genes located on the Y chromosome are exclusively expressed in the testes, or

\footnotetext{
* Correspondence: ri.jansen@ggzingeest.nl

${ }^{\dagger}$ Equal contributors

'Department of Psychiatry, VU University Medical Center, Neuroscience Campus Amsterdam, Amsterdam, The Netherlands

Full list of author information is available at the end of the article
}

are housekeeping genes with X-chromosome homologues that escape $\mathrm{X}$-inactivation [8]. However, genome regulation seems highly sex-specific at secondary epigenetic levels such as DNA methylation [9], DNase hypersensitivity [10], chromatin structure [11] and gene expression $[12,13]$. Thus, a characterization of sex differences in genome regulation by gene expression will contribute to the understanding of the molecular basis of sexual dimorphism.

Animal studies have shown that sex-biased gene expression is highly tissue dependent $[14,15]$ and the evolution rates of sex-biased genes are higher than average [12,16]. Two recent studies in mice reported sex differences in gene expression networks of correlated transcripts $[17,18]$. Surprisingly few studies aimed at identifying and investigating sex-biased genes in humans, and only in small sample sizes $(N<250$ [19-22]). Nonetheless, consistent evidence was obtained for sex-specific gene expression.

Sex-differences in gene expression will depend on the hormonal status of the group considered. For instance, during menopause, much of the female-specific hormone

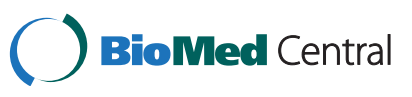


production ceases, with downstream effects on gene expression in adipose tissue [23], monocytes [24], and bone [25]. In women using hormonal contraceptives, containing the hormones estrogen and progesterone, additional differences in gene expression may be evident as well.

For many genes, expression levels are influenced by DNA polymorphisms (eQTLs). Although the sexes do not differ at the autosomal DNA sequence level, sex differences in gene expression may be caused by sex-specific eQTLs [26] (i.e. some SNPs may influence gene expression in one sex, but not in the other).

Here we used microarrays to identify genome-wide sexbiased gene expression in the human peripheral blood transcriptome in a large sample $(N=5241$ subjects $)$ from the Netherlands. The sample size was sufficiently large to account for menopause status and hormonal contraceptive use. The identified sex-biased genes were characterized in terms of enrichment for functional gene ontology (GO) and disease categories, distribution across the autosomes and sex chromosomes, tissue specificity, evolution rates, participation in major gene expression networks and the extent to which sex differences in gene expression were caused by sex-specific eQTLs.

\section{Results}

\section{Sample description}

The sample consisted of 5,241 individuals from the Netherlands Study of Depression and Anxiety (NESDA) and Netherlands Twin Register (NTR) cohorts (Table 1; [27]). Of the women, $22 \%$ were postmenopausal and $31 \%$ used hormonal contraceptives. For all participants, genome-wide gene expression in peripheral blood was assessed using microarrays with 47,122 probe sets targeting 19,250 genes. For each probe set, mixed models including demographic, and several technical covariates were used to test for sex effects (see Methods).

\section{Sex effects on gene expression}

Sex effects on gene expression were determined by comparing men $(N=1,814)$ and premenopausal women who

Table 1 Demographic summary of sample

\begin{tabular}{lcc}
\hline & Female & Male \\
\hline Total \# subjects (after QC) & 3427 & 1814 \\
group (NTR/NESDA) & $2079 / 1348$ & $1147 / 667$ \\
age (mean/sd) & $38.5 / 12.7$ & $38.9 / 13.7$ \\
bmi (mean/sd) & $25.6 / 4.1$ & $24.5 / 4.6$ \\
smoking status (percentage of smokers) & $27.00 \%$ & $32.00 \%$ \\
red blood cell count & $8.3 / 0.6$ & $9.4 / 0.6$ \\
Menopause status females (pre/post) & $2687 / 740$ & \\
Contraceptive pill use (yes/no) & $1093 / 2334$ & \\
\hline
\end{tabular}

The sample consisted of 5241 subjects (after QC) from the Dutch NESDA and NTR cohorts. did not use hormonal contraceptives $(N=1,594)$. When considering 45,418 autosomal transcripts targeting 18,495 genes, 993 transcripts from 582 genes (3.1\% of all autosomal genes measured) were significantly influenced by sex $(p<1.2 \mathrm{e}-6$, Bonferroni corrected at $p<0.05$, FDR $<6 \mathrm{e}-5)$. The percentage of sex-biased genes increased when only genes with a mean expression above a certain threshold were considered. For example, a mean expression threshold of 5 ( $\log _{2}$ (intensity)) resulted in $5.5 \%$ sexbiased genes, and using a threshold of 9 resulted in $13.7 \%$ sex-biased genes (Figure 1A). However, there were several transcripts with low mean expression level but with a high fold change between the sexes (Figure 1B). In order to provide a comprehensive overview, we included all transcripts in the following analyses.

\section{Female-biased versus male-biased genes}

From the sex-biased transcripts on the autosomes, 572 $(57.7 \%)$ were upregulated in females (female-biased genes, Figure 1C), and 421 in males (male-biased genes, Figure 1B). For each sex-biased transcript the $\log _{\mathrm{e}}$ fold change was computed (Figure 1D). For female-biased transcripts the fold change was computed as the mean expression in females/mean expression in males, for male-biased genes we used -mean expression in males/ mean expression in females. Most absolute $\log _{e}$ fold changes were smaller than 0.08 (99\%), for 22 transcripts the absolute $\log _{\mathrm{e}}$ fold change was larger than 0.08 (6 femalebiased, targeting the genes ADM, CREB5, CNTNAP3, C9orf84, SORCS2 and GPR109A and 14 in men (KANK2, CTSG, MPO, BPI, GPER, DEFA4, EPB49, C19orf62, ERG, LCN2, CEACAM8, LTF, FECH and LTBP1), see Additional file 1 for sex-biased genes and corresponding fold changes and $p$-values).

On the X chromosome, 1643 transcripts from 739 genes were measured. Out of these, 127 transcripts from 51 genes were sex-biased; 103 (from 38 genes) were femalebiased, and 24 (from 13 genes) male-biased. Seventeen of the corresponding $\log _{\mathrm{e}}$ fold changes were larger than 0.08 (targeting the genes EIF1AX, PRKX, KDM5C, ZFX, KDM6A, XIST, VSIG4, TSIX and SCARNA9L). Only the $\log _{\mathrm{e}}$ fold changes of the genes XIST and TSIX were larger than 0.5 . Of the 63 transcripts targeting 26 genes on the Y chromosome, 48 transcripts from 16 genes had expression levels in men that were higher than the noise measured in women; 12 transcripts had a $\log _{\mathrm{e}}$ fold change larger than 0.5 , targeting the genes EIF1AY, DDX3Y KDM5D, CYorf15B, CYorf15A and UTY.

\section{Genomic location of sex-biased genes}

For each chromosome we tested whether the genes on that chromosome enriched the sex-, male- or femalebiased genes. At the autosomes, the percentage of sexbiased genes differed only slightly between chromosomes, 


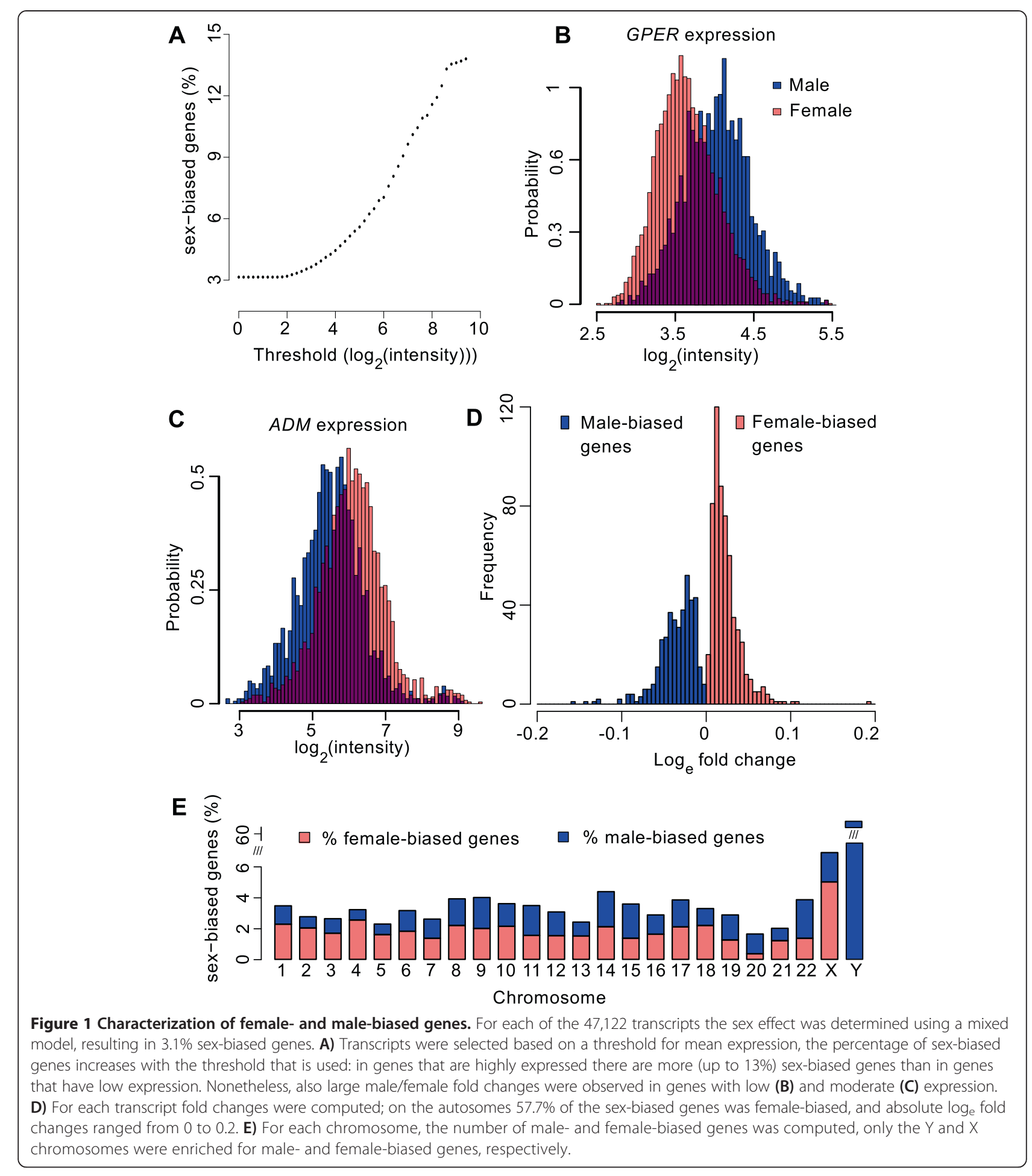

ranging from $1.6 \%$ on chromosome 20 to $4.2 \%$ on chromosome 14 (Figure 1E); none of the autosomes enriched the sex-biased genes ( $p>0.05$, Fisher's exact test). The distribution of the male- and female-biased genes over the autosomes was more variable, ranging from $0.4 \%$ at chromosome 20 to $2.3 \%$ at chromosome 18 (female- biased genes), and from $0.7 \%$ at chromosome 4 to $2.4 \%$ at chromosome 22 (male-biased gene), however none of the autosomes enriched female or male-biased genes. As expected, female-biased genes were enriched for genes at the $\mathrm{X}$-chromosome (5.1\%), and male-biased genes for genes at the Y chromosome (61\%). 


\section{Gene ontology (GO) analysis of sex-biased genes}

Female-biased genes were enriched for 52 biological process GO categories (BPGO) $(p<0.01$, Bonferroni correction, see Additional file 2 for significant GO categories and female-biased genes therein), with as top hit immune system process $(31.6 \%$ of female-biased genes are in this category, $p<1 \mathrm{e}-25)$. Significant subcategories included response to cytokine stimulus $(11.3 \%, p<1 \mathrm{e}-10)$, response to type 1 interferon $(3.6 \%, p<1 \mathrm{e}-7)$ and lymphocyte differentiation (5.6\%, $p<1 \mathrm{e}-5)$. Male-biased genes were not enriched for any BPGO category. The femalebiased genes were enriched for 7 cellular component GO (CCGO) categories (top hits are cell surface $(8.3 \%$, $p<1 \mathrm{e}-6)$ and integral to membrane $(31.3 \%, p<1 \mathrm{e}-5))$. Male-biased genes were enriched for 11 CCGO categories, with as top hit cytoplasm $(73.4 \%, p<1 \mathrm{e}-10)$ and significant subcategory lysome $(6.4 \%, p<1 \mathrm{e}-4)$. In Additional file 3 the hierarchical network structure of the significant GO categories is visualized.

\section{Ingenuity pathway analysis (IPA) of sex-biased genes} From the IPA biological functions, autoimmune disease $(22 \%$, FDR $<1 \mathrm{e}-11$ (Fisher's exact test)) and rheumatoid arthritis $(16 \%$, FDR $<1 \mathrm{e}-11$ (Fisher's exact test)) enriched the female-biased genes most significantly, among 21 other autoimmune diseases (Additional file 4). The upstream regulator lipopolysaccharide (LPS, 26\%, $p<1 \mathrm{e}-27$ (uncorrected $p$-value Fisher's exact test)) was most strongly associated with the female-biased genes, among many other regulators (Additional file 5) such as estradiol (18\%, $p<1 \mathrm{e}-9$ (uncorrected $p$-value Fisher's exact test)). Male-biased genes were most significantly enriched for genes linked to renal cancer $(9 \%$, FDR $<1 \mathrm{e}-5$ (Fisher's exact test), Additional file 6). There were only few malebiased genes influenced by the same upstream regulators (Additional file 5, top hits were GATA (3\%, $p<1 \mathrm{e}-5$ (Fisher's exact test)) and HIPK2 (2\%, $p<1 \mathrm{e}-4$ (Fisher's exact test))).

\section{eQTL analysis of sex-biased gene expression}

eQTL analysis was performed using two sample subsets of 1523 men and 1373 premenopausal women who did not take hormonal contraceptives, for which genomewide SNP and gene expression data were available (see Methods). For each of the 993 autosomal sex-biased transcripts, eQTLs were computed for men and women separately. At a FDR of 0.01 there were 7978 cis eQTLs $(p<6 \mathrm{e}-05)$ and 514 trans eQTLs $(p<2 \mathrm{e}-09))$ for men, and 6731 cis eQTLs $(p<5.2 \mathrm{e}-05)$ and 197 trans eQTLs $(p<1.8 \mathrm{e}-09))$ for women. For the pooled eQTLs (9659 cis, 545 trans eQTLs) genotype-sex interactions were assessed using a mixed model that included data from men and women. At a FDR of 0.05 no significant genotype-sex interactions were observed.

\section{Sex-biased genes highly enrich modules of correlated transcripts}

Weighted Gene Co-Expression Network Analysis (WGCNA) [28] was used to identify modules of correlated transcripts, for men and women separately. Both analyses resulted in 9 modules with $>70 \%$ transcript overlap between the male and the corresponding female module for 8 of the 9 modules (Additional file 7). One module had only $\sim 40 \%$ overlap. Thus, gene expression correlation structure is similar between men and women, and here we focus on properties of the intersection of the overlapping modules. Interestingly, 7 of these intersected modules were highly enriched with femalebiased or male-biased genes. There were three modules with more than 30\% male-biased genes, and two modules with more than $30 \%$ female-biased genes. The modules were highly enriched for several GO terms (Additional file 7). We calculated the pairwise transcript correlations within each intersected module or men and women separately. For two modules containing malebiased genes the correlations were significantly stronger in males than in females $(76 \%$ of the correlations were stronger in module \#6, and 92\% in module \#9, Figure $2 \mathrm{~A}$ $\& \mathrm{~B}$ respectively). Thus, these modules contained around $30 \%$ of male-biased genes, but also the majority (> 75\%) of the interactions in the module were stronger in males compared to females.

\section{Evolution rates of sex-biased genes}

To test whether sex-biased genes have evolved faster than non sex-biased genes, we tested for enrichment in two sets of genes that were previously identified as rapidly evolving: 244 genes from the Human PAML Browser [29] and 40 genes from a study comparing human and chimpanzee genomes [30]. Sex-biased, male-biased and femalebiased genes were not enriched for any of the two gene sets (Fisher's exact test, $p>0.05$ ). Next, we tested whether $\mathrm{dN}, \mathrm{dS}$ and $\mathrm{dN} / \mathrm{dS}$ (the evolution rates) as provided by [31] were different in sex-biased, male-biased and femalebiased genes compared to non sex-biased genes, but found no significant differences (all $p>0.05$, Wilcoxon rank test).

\section{Tissue specificity of sex-biased genes}

We downloaded analysis results of two human studies that identified sex-biased genes in muscle [22] and in liver [21]. In muscle, 63 sex-biased genes were identified on the autosomes which were enriched with the sexbiased genes we identified ( 8 genes identified in both tissues, $p<0.01$ (Fisher's exact test), Additional file 8). On the $\mathrm{X}$ chromosome 5 genes were identified as sexbiased in muscle, of which 4 were also identified in blood ( $p<0.001$ (Fisher's exact test), Additional file 8). In liver, 862 sex-biased genes were identified on the autosomes which were enriched with the sex-biased genes 

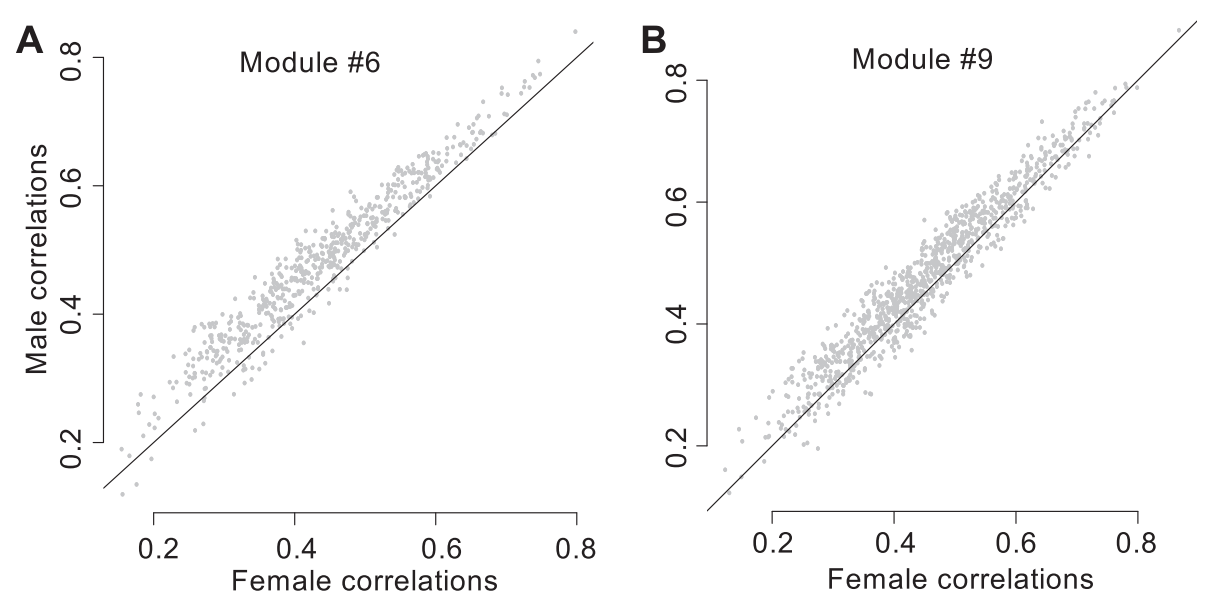

Figure 2 Between transcript correlations are higher in males than in females for $\mathbf{2}$ modules. WGCNA (Weighted Gene Co-Expression Network Analysis) resulted in 9 modules with correlated transcripts, two of which were highly enriched for female-biased genes, and 3 for male-biased genes. From the latter three, two modules contained genes from which the pair-wise correlations were stronger in males compared to females. A) Module \#6 contained 45 genes, $76 \%$ of the correlations computed in males ( $y$ axis) were larger than those computed in females ( $x$ axis). B) Module \#9 contained 35 genes, 92\% of the correlations computed in males (y axis) were larger than those computed in females ( $x$ axis).

we identified (36 genes identified in both tissues, $p<0.05$ (Fisher's exact test), Additional file 8). On the X chromosome 50 genes were identified as sex-biased in liver, of which 18 were also identified in blood ( $p<1 \mathrm{e}-9$ (Fisher's exact test), Additional file 8).

\section{Sex-biased genes in postmenopausal and hormonal contraceptive using women}

To examine whether sex differences in gene expression depend on hormonal status, sex effects were computed by comparing men $(N=1,814)$ with postmenopausal women $(N=740)$ and women using hormonal contraceptives (HC women, $N=1,093)$. On the autosomes, there were 697 transcripts differentially expressed between postmenopausal women and men. From these 697 transcripts (369 female-biased and 328 male-biased) 236 overlapped with the 993 sex-biased transcripts identified in non-hormonal contraceptives using premenopausal (NHC) women. When comparing the $\mathrm{HC}$ women with men, a much larger number of 2,125 differentially expressed transcripts were identified (1,157 female-biased, 968 in male-biased). From these transcripts, 755 were overlapping with the 993 sex-biased transcripts identified in NHC women. For the 933 transcripts identified in $\mathrm{NHC}$ women, $\log _{\mathrm{e}}$ fold changes were computed for the difference between each of the three groups of women (NHC, HC, postmenopausal) compared to men. When comparing these fold changes between postmenopausal and $\mathrm{NHC}$ women, it became clear that most of the fold changes have the same sign ( $85 \%$ in total, $99 \%$ of the negative fold changes) but that the fold changes in NHC women are larger than those in postmenopausal women for $80 \%$ of the transcripts (Figure 3A). Also the fold changes of $\mathrm{NHC}$ women and $\mathrm{HC}$ women often have the same sign (96\%), and the fold changes of $\mathrm{HC}$ women were often larger than those observed in NHC women (66\% of all fold changes, $88 \%$ of the negative fold changes, Figure $3 \mathrm{~B})$. This shows that many gene expression differences between women and men become smaller when women reach menopause, and are larger when women use hormonal contraceptives, which reinforces the role of estrogen in regulating sex-biased genes.

\section{Age specific sex effects on gene expression}

Age has a strong influence on gene expression [32]. To examine whether sex effects on gene expression are agerange specific, we separately analyzed the data for three age groups (men versus premenopausal women who did not use hormonal contraceptives, age ranges 17-30 ( $N=$ $1047), 31-40(N=1191)$ and $41-88(N=1170))$. In these 3 age groups we identified 49, 103 and 34 autosomal sex-biased genes respectively $(p<1.2 \mathrm{e}-6$, Additional file 9 ), which overlapped for $>98 \%$ with the sex-biased genes identified in the total sample (with same direction of effect). The three sets of sex-biased genes identified in these age groups overlapped to a lesser extent with each other ( $>38 \%$, Additional file 9). However, the fold changes between men and women of the sex-biased genes identified in the total sample were highly concordant between age ranges (Additional file 10), suggesting that the identified sex effects occur at all ages, but that some effects may be stronger at a certain age or may not have been identified due to reduced power in the smaller groups of selected ages. 

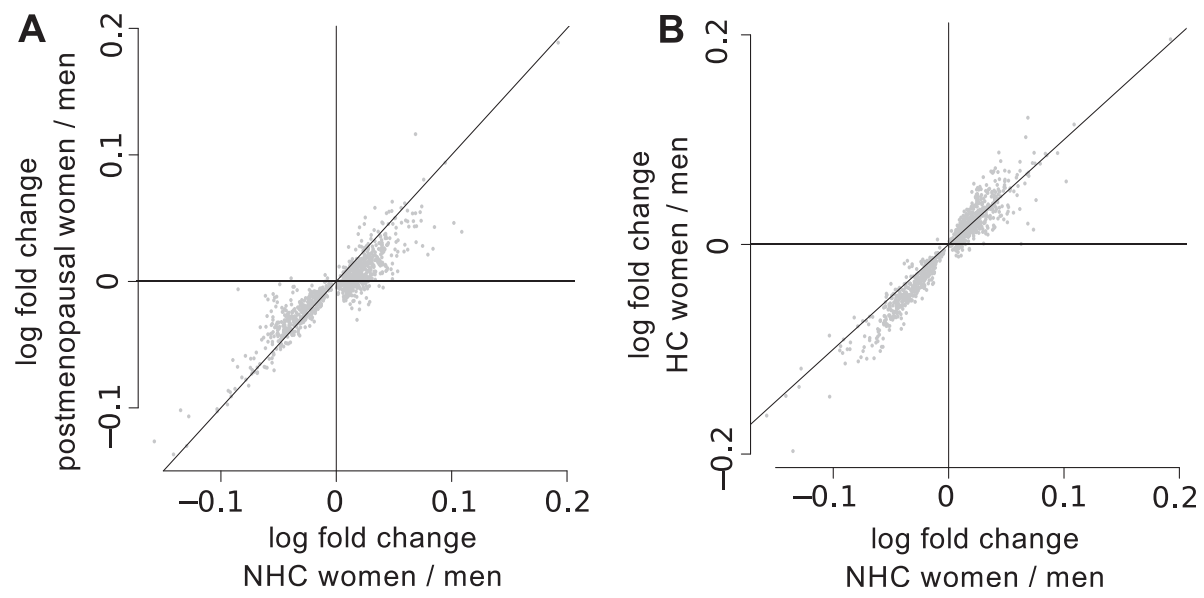

Figure 3 Sex-differences in gene expression are increased by the use of hormonal contraceptives, and decreased during menopause. Women were divided in three groups: postmenopausal, hormonal contraceptive using (HC), and non hormonal contraceptive using (NHC) women. For the 993 sex-biased transcripts identified in the comparison between males and NHC women, fold changes were computed for the difference between the three groups of women and the men. Positive fold changes are from female-biased genes, negative fold changes correspond to male-biased genes. A) Fold changes are for $80 \%$ larger in NHC women as compared to postmenopausal women. B) Fold changes in HC women are for $66 \%$ larger than those observed in NHC women, and the negative fold changes (male-biased genes) were for $88 \%$ larger in $\mathrm{HC}$ women.

\section{Discussion}

At the DNA autosomal sequence level sexes do not differ, as established by a well-powered meta-analysis [33], suggesting an important role for higher molecular levels, such as the transcriptome, in the manifestation of sexual dimorphisms. Indeed, animal studies have shown that the transcriptome is highly differential between sexes $[14,15,34,35]$. In humans, gene expression differences have been reported in liver [21], lymphoblastoid cell lines [19,20], and muscle [22], but only in studies with relatively small sample sizes $(N<250)$. Here we analyzed the sex differences in the peripheral blood transcriptome by assessing 47,122 probe sets targeting 19,250 genes genome wide in a well-characterized large Dutch cohort $(N=5,241)$ taking into account the impact of hormonal contraceptive use and menopausal status in women.

\section{Number of female- and male-biased genes}

On the autosomes, we identified 582 genes (3.1\% of all genes measured) that were differentially expressed between men and premenopausal women not using hormonal contraceptives. Of these genes, $57.7 \%$ were female-biased. The autosomes had rather similar proportions of sex-biased genes indicating the sex-biased genes can be found equally frequent across the entire genome, as opposed to what was found in liver [21] where several chromosomes enrich sex-biased genes. It is important to note that the filter criteria used for selecting probe sets highly influences the number of sex-biased genes; the percentage of sex-biased genes increased with the threshold for mean expression level from $3.1 \%$ up to $13.7 \%$. Importantly, we have shown that hormonal contraceptives and menopause status, which were not taken into account in previous studies in humans, highly influence the number and effect sizes of sex differences in gene expression. Although it has been indicated that the percentage of sexbiased genes in non-human vertebrates is highly tissue dependent (e.g. ranging from $13.6 \%$ in the brain to $72 \%$ in the liver $[14,15]$ ), our described range of $3.1-13.7 \%$ for sexbiased genes is comparable to that found in human liver $(3.7 \%,[21])$. Peripheral blood consists of a mixture of blood cell types (the main types are lymphocytes, neutrophiles and monocytes), hence the sex differences we identified must either be present in all subcell types or, when present in only one cell type, strong enough to be observed in the accumulative measurement. By stratifying the sample into three age groups we showed that the size of the sex effects may be age dependent for some genes, but the direction of the effects are highly concordant between age groups.

We found a significant but small overlap of sex-biased autosomal genes identified in peripheral blood with those previously identified in muscle or liver, further confirming substantial tissue specificity of sex-biased genes. Across tissue circulating exosomes contain RNA and could contribute to the overlapping expression profiles between muscle, liver and blood [36]. Sex-biased X chromosome genes showed must larger overlap between tissues, indicating that escape from X-inactivation is highly similar between tissues. Previous studies have reported that sexbiased genes may evolve more rapidly than average in vertebrates [12], human brain [37] and liver [21]. However, sex-biased genes in the peripheral blood transcriptome identified in our study did not include enrichment of fast evolving genes. In women, most genes on one $\mathrm{X}$ chromosome are not expressed due to $\mathrm{X}$ chromosome inactivation 
[38]. Some genes escape X-inactivation and are expressed from both X chromosomes [7]. We showed that in peripheral blood the $\mathrm{X}$ chromosome is enriched for femalebiased genes; $5.1 \%$ of the genes measured on the $\mathrm{X}$ chromosome are female-biased. This percentage, however, is only slightly higher than the average percentage identified at the autosomes (3.1\%), which shows a major role of autosomal genes in sex-specific gene expression.

\section{The role of estradiol in gene expression sex differences}

Estrogen is the primary female sex hormone and estrogenic activity is present at about two fold increased concentration in women as compared to men. Estradiol, the predominant estrogen in terms of absolute serum levels, activates estrogen receptors that bind to DNA sequences to activate or suppress gene expression, and many efforts have been made to find its target genes (up to 5000) in MCF-7 cancer cell line [39-41] because of its role in breast cancer [42]. Here we show that in peripheral blood $18 \%$ of the identified sex-biased genes are known to be regulated by estradiol, and several additional findings suggest that the sex difference in estrogen levels underlie multiple sex differences in gene expression. First, from the 20 genes with high male/female fold changes, 7 are involved in common diseases and influenced by estrogen; GPER (g protein-coupled estrogen receptor-1, related to cancer [43]), $A D M$ (coding for the peptide adrenomedulin, the main vasodilatory peptide involved in cardiovascular disease [44-46], LTF (lactoferrin, essential for the innate immune system and involved in cancer $[47,48]$ ), LCN2 (lipocalin-2, innate immune system and cancer related [49]), MPO (myeloperoxidase), a biomarker for cardiovascular disease risk [50], ERG (Ets Related Gene, proposed as a mediator of estrogen effect on prostate cancer [51], LTBP1 (latent-transforming growth factor beta-binding protein 1, linked to coronary heart disease [52]. This suggests that these genes mediate the effect of estrogen and thereby may contribute to the sex differences in the related diseases. Second, we showed that the sex differences in gene expression depend largely on the hormonal status of the subgroup of women considered. In postmenopausal women, in which estradiol levels are similar to those in men, we identified fewer sex-biased genes with smaller effect sizes as compared to premenopausal women. In hormonal contraceptive using women, with increased estradiol levels, we identified more sex-biased genes and larger effect sizes as compared to women not using hormonal contraceptives. Interestingly, the change in effect size was present for more than $65 \%$ of the female-biased genes, and for more than $85 \%$ of the male-biased genes. This gives an indication of the amount of sex-biased genes affected by estradiol, which is much higher than currently known from literature (IPA, 15\% of sex-biased genes are known to be regulated by estradiol). In liver, sex differences in gene expression are mainly caused by sex-specific growth hormone secretion [21,53]. Growth hormones are regulated by estrogen $[54,55]$, hence the effect of estrogen on sex-specific gene expression in peripheral blood may also be mediated by growth hormone secretion.

\section{Immune system processes predominant in female-biased genes}

The immune system function is known to be different between sexes; women produce more vigorous immune reactions and are more prone to autoimmune diseases [56]. Here we identified a large number of genes that potentially contribute to the immune system sex differences; $31.6 \%$ of female-biased genes are in the GO category immune system process. From the 95 femalebiased genes linked to the immune system, 45 are regulated by estradiol, which confirms the role of estrogen in the sex-specific immune system functioning [57]. Most interestingly, Ingenuity Pathway Analysis revealed that female-biased genes are highly enriched for genes involved in the toll-like receptor (TLR4 and TLR3 pathways, known as LPS and poly I:C response patterns) driven innate immune defense, suggesting some intrinsic innate immune activity sex differences. Increased female expression of immunoglobulin is reflective of concomitant more active humoral immune activity. These functions are compatible with an activated leukocyte, cytokine production and type 1 interferon activity observed in the GO enrichment analysis and might explain why women are more resistant to certain infections, and suffer a high incidence of autoimmune diseases compared to men [2]. For example, rheumatoid arthritis occurs almost twice as often in women as in men [58]. Female-biased genes were enriched for genes linked to rheumatoid arthritis, including the gene $I L 6 R$, which is a well-known target in rheumatoid arthritis treatment [59]. The identified female-biased genes provide a framework for future research to unravel the mechanism of sex-biased immune regulation and autoimmune diseases.

\section{Annotation of male-biased genes}

Surprisingly, male-biased genes were not enriched for GO categories, and thus serve a wide variety of biological functions. In IPA, however, male-biased genes were most significantly enriched for genes linked to renal cancer, including the well established renal cancer gene CSF1R [60]. It is notable that a recent meta-analysis on sex differences in renal cell cancer presentation and survival showed a ratio of 1.65 of renal cell carcinoma for males compared to females [61]. The cellular component GO categories indicate the part of a cell at which a gene product is located. Topographical categorization revealed that male-biased 
gene products occur more often intracellularly, in particular at the cytoplasm, whereas female-biased genes occur more often integral to the membrane.

\section{Sex-specific eQTLs do not underly sex-biased gene expression}

A previous study (in a smaller sample than the current one) showed that a substantial amount of eQTLs is sexspecific, but not for eQTLs from genes with sex-biased expression [26]. Here we confirm this finding by showing that for the sex-biased genes there were no significant eQTL-sex interactions. This shows the importance of other factors, such as estradiol and other hormones, in causing gene expression sex differences.

\section{Sex-biased genes in modules of correlated transcripts}

WGCNA analyses resulted in highly similar modules of correlated transcripts for men and women, similar to findings in mice [18]. The 9 modules were highly enriched for male or female-biased genes, indicating that sex-biased genes play an important role in the major gene expression networks. Module \#2 and \#3 contained each more than 30\% female-biased genes and were enriched for the GO category immune system response, which shows that immune system genes operate in correlated groups that are partially sex-biased. Module \#9 contained $31.4 \%$ male biased genes, enriched the GO category immune response (37\%) and contained 92\% stronger pairwise correlations in men than in women. This module contained the interleukin receptor IL2B gene, and IPA analysis showed that 11 of the 35 genes in this module are known to be regulated by the cytokine IL2, and 16 of them are related to cancer (Additional file 11) including the female-biased genes PRF1 and GZMH essential for natural killer (NK)-cell cytotoxicity [62,63]. Module \#6 contained $37.8 \%$ male-biased genes, was enriched for the GO term coagulation (50\%) and $76 \%$ of the pairwise correlations in this module are higher in men than in women. IPA analysis shows that from this module 16 genes are regulated by TGFB1 (Additional file 11), and 17 genes are related to heart or vascular disease, including the male-biased genes PTGS1 (coding for COX-1, which is inhibited by aspirin [64] that has a protective effect on cardiac events [65]), ITGA2B, ITGB3, F13A and GP1BA which are candidate stroke risk genes [66]. This suggests that the modules \#9 and \#6 may play a role in the sex differences in cancer and cardiovascular disease, respectively.

\section{Conclusions}

We showed that sex-biased genes occur in large numbers throughout the human peripheral blood transcriptome, suggesting an important role of sex-specific gene expression in sexual dimorphisms. Estrogen appears to be a key regulator of sex-biased genes, also shown by the effect of menopause and hormonal contraceptives on gene expression sex differences. Sex-biased genes are highly enriched with genes linked to common diseases and may contribute to sex-differences in these diseases. Understanding the molecular mechanisms behind sex inequalities can lead to new insights into sex-specific pathophysiology and treatment opportunities.

\section{Methods}

\section{Subjects}

The two parent projects that supplied data for this study are large-scale longitudinal studies: the Netherlands Study of Depression and Anxiety (NESDA) [67] and the Netherlands Twin Registry [68]. NESDA and NTR studies were approved by the Central Ethics Committee on Research Involving Human Subjects of the VU University Medical Center, Amsterdam (IRB number IRB-2991 under Federalwide Assurance 3703; IRB/institute codes, NESDA 03-183; NTR 03-180), and all subjects provided written informed consent. The sample consisted of 5391 subjects (before QC), 3327 participants from NTR (2 MZ triplets, $708 \mathrm{MZ}$ twin pairs, $658 \mathrm{DZ}$ twin pairs, 338 siblings from these twins and 251 unrelated individuals) and 2064 unrelated participants from NESDA. The age of the participants ranged from 17 to 88 years (mean 38, SD 13) and $65 \%$ of the sample was female. As part of the NESDA and NTR biobank protocols, data on menopause status and medication use, including hormonal contraceptives were collected in all participants.

\section{Blood sampling, RNA and DNA extraction}

The NTR and NESDA blood sampling and RNA extraction procedures have been described in detail previously [69,70]. In short; for NTR, venous blood samples were drawn between 0700-1100 after an overnight fast and usually in the subjects' homes. Within 20 minutes of sampling, heparinized whole blood was transferred into PAXgene Blood RNA tubes (Qiagen) and stored at $-20^{\circ} \mathrm{C}$. The PAXgene tubes were shipped to the Rutgers University Cell and DNA Repository (RUCDR), USA. Average time between blood sampling and RNA extraction was 211 weeks (included in mixed model for gene expression). Upon registration of samples, RNA was extracted using Qiagen Universal liquid handling system (PAXgene extraction kits as per the manufacturer's protocol).

From the NESDA subjects, serial venous whole blood samples were obtained (8-10 AM, after overnight fasting) in one 7-mL heparin-coated tube (Greiner Bio-One, Monroe, North Carolina). Between 10 and 60 min after blood draw, $2.5 \mathrm{~mL}$ of blood was transferred into a PAXgene tube (Qiagen, Valencia, California). This tube was kept at room temperature for a minimum of 2 hours and then stored at $-20^{\circ} \mathrm{C}$. Average time between blood sampling and RNA extraction was 113 weeks (included 
in mixed model for gene expression). Total RNA was extracted at the VU University Medical Center (Amsterdam) according to the manufacturer's protocol (Qiagen) as described previously [70].

For both NESDA and NTR samples high molecular weight genomic DNA was isolated from frozen blood in EDTA tubes using Puregene DNA isolation kits (Qiagen).

\section{Gene expression measurements}

Gene expression assays were conducted at the Rutgers University Cell and DNA Repository (RUCDR, http:// www.rucdr.org). RNA quality and quantity was assessed by Caliper AMS90 with HT DNA5K/RNA LabChips. RNA samples that showed abnormal ribosomal subunits in the electropherograms were removed. NTR and NESDA samples were randomly assigned to plates with seven plates containing subjects from both studies to better inform array QC and study comparability. For cDNA synthesis, $50 \mathrm{ng}$ of RNA was reverse-transcribed and amplified in a plate format on a Biomek FX liquid handling robot (Beckman Coulter) using Ovation Pico WTA reagents per the manufacturer's protocol (NuGEN). Products purified from single primer isothermal amplification (SPIA) were then fragmented and labeled with biotin using Encore Biotin Module (NuGEN). Prior to hybridization, the labeled cDNA was analyzed using electrophoresis to verify the appropriate size distribution (Caliper AMS90 with a HT DNA $5 \mathrm{~K} /$ RNA LabChip). Samples were hybridized to Affymetrix U219 array plates (GeneTitan) to enable highthroughput gene expression profiling of 96 samples at a time. The U219 array contains 530,467 probes for 49,293 transcripts. All probes are 25 bases in length and designed to be "perfect match" complements to a designated transcript. Array hybridization, washing, staining, and scanning were carried out in an Affymetrix GeneTitan System per the manufacturer's protocol.

\section{Genome-wide SNP measurements and QC}

Genotyping was conducted using Affymetrix GenomeWide Human SNP Array 6.0 containing 931,946 SNPs, per the manufacturer's protocol. The resulting data were required to pass standard Affymetrix QC metrics (contrast $\mathrm{QC}>0.4$ ) before further analysis. SNP QC included removal of SNPs for non-unique mapping of probe sequences to NCBI Build 37/UCSC hg19, low minor allele frequency $(<0.005)$, substantial deviation from HapMap3 CEU founder allele frequencies, deviation from Hardy-Weinberg equilibrium $\left(\mathrm{p}_{\mathrm{HWE}}<1 \times 10^{-8}\right)$, and high missingness (>0.05). After genotyping QC, $666 \mathrm{~K}$ autosomal SNPs were available. Subjects were eliminated from analysis for high missingness $(>0.05)$, outlying genome-wide homozygosity or ancestry, discrepant genetic and phenotypic sex, or twin relatedness not consistent with monozygosity or dizygosity.

\section{Gene expression QC}

Gene expression data were required to pass standard Affymetrix QC metrics (Affymetrix expression console) before further analysis. Probes were removed when their location was uncertain or if their location intersected a polymorphic SNP (dropped if the probe oligonucleotide sequence did not map uniquely to hg19 or if the probe contained a polymorphic SNP based on HapMap3 and 1000 Genomes project data). Expression values were obtained using RMA normalization implemented in Affymetrix Power Tools (APT, v 1.12.0). First, 70 samples with array results inconsistent with the phenotypic database were removed (inconsistent sex based on chr X and chr Y probe sets). Second, we used the pairwise correlation matrix of expression profiles across all arrays for additional QC. These quantities were expressed in terms of median absolute deviations to provide a sense of scale. We used:

$$
D_{i}=\frac{\left|r_{i}-r\right|}{\operatorname{median}\left(\left|r_{k}-r\right|\right)_{k=1 \ldots N}}
$$

With $r_{i}$ the average of correlations for sample $i$, and $r$ the average of all correlations. Larger values of $D$ corresponded to poor quality; 80 samples with $D>5$ were removed, decreasing the final number of subjects to 5,241 .

\section{Mixed models for gene expression}

Linear mixed models allow for the correction for the presence of twin families in a sample [71]. For each of the 47,122 probe sets a mixed model was fit with gene expression as dependent variable. Independent model covariates were selected based on significance of the variable in the fitted mixed models. Several covariates that did not come out significantly were not included in the final model (alcohol use, education level, time between RNA amplification and RNA fragmentation, time between RNA fragmentation and RNA hybridization). Inclusion of depression status and psychotropic medication use as covariates in the mixed model did not affect the principle findings. Fixed effect covariates included in the final model were sex, age, body mass index (BMI, weight/height 2 in $\mathrm{kg} / \mathrm{m}$ ), smoking status (yes/no current smoking), D (see above), hemoglobin ( $\mathrm{mmol} / \mathrm{L})$, group (NTR or NESDA), time of blood sampling, month of blood sampling, time between blood sampling and RNA extraction, and the time between RNA extraction and RNA amplification. Random effects were plate, well, family ID and zygosity (one factor for each monozygotic twin pair, for each other individual different factors [71]). In Additional file 12, for each of the variables the amount of probe sets for which the variable was significant is denoted. Mixed models and resulting p-values were computed using the $\mathrm{R}$ function lmer from the package lme4. 


\section{eQTL analysis}

eQTL analysis was first performed in a screening step by MatrixeQTL [72]. Prior to eQTL analysis for each gene expression probeset the data was transformed into a normal distribution using an inverse quantile normal transformation. Genotypes were coded as 0,1 or 2 and for each SNP-transcript pair a linear regression model was fitted including the covariates sex, age, body mass index, smoking status, D (see above), hemoglobin ( $\mathrm{mmol} / \mathrm{L})$, group (NTR or NESDA), time of blood sampling, month of blood sampling, time between blood sampling and RNA extraction, time between RNA extraction and RNA amplification, plate and well plus three principle components (PCs) from the genotype data [73] and 5 PCs from the transformed expression data. Cis-eQTLs are transcriptassociated SNPs with distance $<1 \mathrm{Mb}$ of transcript site. The trans-eQTLs are the complementary set of SNPs. In the screening step, males and woman were screened using MatrixeQTL as if the individuals were all un related. Benjamini-Hochberg q-value estimation was performed separately for cis- and trans-eQTLs. For each of the 993 autosomal sex-biased transcripts eQTLs were selected for men and women separately, and then pooled. For these eQTLs genotype-sex interactions were assessed using the full mixed model that included both men and women, with as independent variables genotype, sex, their interaction and the other covariate also used in the mixed model for gene expression (see above).

\section{GO category enrichment}

To test whether Gene Ontology [74] categories enriched sex-biased genes we used hypergeometric tests implemented in BINGO software [75]. The reference gene set consisted of all genes measured by the U219 microarrays.

\section{WGCNA}

The correlation structure of gene expression was examined using unsigned co-expression networks constructed using the WGCNA package in R [28]. Of all 47,122 probes a single probe of highest mean expression per gene was selected to be included in the network analysis using the CollapseRows function in WGCNA, resulting in the inclusion of 19,249 genes in the network. The choice of the probe of highest mean expression per gene has been shown to yield robust analysis across data sets [76]. The network construction for each entire data set was performed in a single block of maximum size 20,000 genes using the blockwiseModules function in WGCNA [28]. Using this block size in WGCNA ensured the theoretical advantage that the genes did not have to be pre-clustered by WGCNA. The network adjacency matrix is the gene pair-wise correlation matrix raised to the power of 6 , chosen based on the scale-free topology criteria [77]. Rather than just using adjacency weights between genes, the topological overlap measure (TOM) is computed from the adjacency matrix. For each pair of genes, TOM is the adjacency weights of all the paths between the genes of length at most two (i.e. the genes are directly connected or have one gene between them) scaled by the minimum connectivity of the either gene. The topological overlap dissimilarity, defined as 1-TOM, is used for the average linkage hierarchical clustering algorithm. The resultant clustering tree is used to define the modules from its branches using the hybrid dynamic tree cutting algorithm [28]. The minimum module size was set to 30 and the cut-off for merging modules was set to 0.25. Each module is then characterized by its eigengene, the first principal component of the module expression data, which accounts for the greatest variation of the expression levels in the module. Genes were removed from modules if the correlations between their expression values and the module eigengenes were too low (less than 0.3). Modules were merged if the correlation between their eigengenes was high.

\section{Availability of supporting data}

Gene expression data used for this study will be available at dbGaP, accession number phs000486.v1.p1 (http://www. ncbi.nlm.nih.gov/projects/gap/cgi-bin/study.cgi?study_id=p hs000486.v1.p1).

\section{Additional files}

Additional file 1: Sex-biased genes and corresponding p-values and $\log _{\mathrm{e}}$ fold change.

Additional file 2: Significant GO categories for female-biased genes.

Additional file 3: Hierarchical structure of Gene Ontology categories enriching male or female-biased genes. $\mathrm{GO}$ enrichment analysis for female (A, B) and male-biased genes (C) in the main categories biological process (A, C) and cellular component (B). GO categories are represented as circles, size of circle shows total amount of genes in this category, color of circle codes for $\mathrm{p}$-value for enrichment (the color white means not significant). An arrow pointing from category A to category $B$ means that $B$ is a subcategory of A. GO categories containing few genes with no further subcategories often occur at the outside of the network, and provide the most specific classification, such as categories response to type 1 interferon, lymphocyte differentiation, anti-apoptosis and lysome.

Additional file 4: Autoimmune diseases enriching female-biased genes.

Additional file 5: Upstream regulators associated with sex-biased genes.

Additional file 6: Male biased genes associated with renal cancer. Additional file 7: Characterization of modules.

Additional file 8: Sex-biased genes identified in multiple tissues. Additional file 9: Sex-biased genes in three age ranges.

Additional file 10: Fold changes of sex-biased genes in three age groups. Subject were divided in three age groups: 18-30, 31-40, and 41-88. For the 993 sex-biased transcripts identified in the full sample, fold changes between men and women were computed within the three groups and plotted against each other. The figures show that the fold changes are highly concordant between age ranges.

Additional file 11: Characterization of genes in Modules \#6 and \#9. Additional file 12: Covariates selected for mixed model. 


\section{Abbreviations}

GO: Gene ontology; BPGO: Biological process GO; CCGO: Cellular component GO; WGCNA: Weighted gene co-expression network analysis; HC women: Women using hormonal contraceptives; NHC women: Non-hormonal contraceptives using women; IPA: Ingenuity pathway analysis.

\section{Competing interests}

The authors declare that they have no competing interests.

\section{Author's contributions}

RJ and SB performed analysis. AB and JT generated molecular data. GW, VM and GVG provided data management support, JJH, YM and HM provided genotype data QC. RJ, WP JV, CLV, EG, JS, FW, PS, DB and BP wrote the manuscript. All authors read and approved the final manuscript.

\section{Acknowledgements}

The Netherlands Study of Depression and Anxiety (NESDA) and the Netherlands Twin Register (NTR) were funded by the Netherlands Organization for Scientific Research (MagW/ZonMW grants 904-61-090, 985-10-002,904-61-193,480-04-004, 400-05-717, 912-100-20; Spinozapremie 56-464-14192; Geestkracht program grant 10-000-1002); the Center for Medical Systems Biology (NWO Genomics), Biobanking and Biomolecular Resources Research Infrastructure, VU University's Institutes for Health and Care Research and Neuroscience Campus Amsterdam, NBIC/BioAssist/RK (2008.024); the European Science Foundation (EU/QLRT2001-01254); the European Community's Seventh Framework Program (FP7/2007-2013); ENGAGE (HEALTH-F4-2007-201413); and the European Science Council (ERC, 230374). Gene-expression data was funded by the US National Institute of Mental Health (RC2 MH089951) as part of the American Recovery and Reinvestment Act of 2009.

\section{Author details}

${ }^{1}$ Department of Psychiatry, VU University Medical Center, Neuroscience Campus Amsterdam, Amsterdam, The Netherlands. ${ }^{2}$ Department of Biological Psychology, VU University Amsterdam, Neuroscience Campus Amsterdam, Amsterdam, The Netherlands. ${ }^{3}$ Department of Biostatistics, University of North Carolina, North Carolina, USA. ${ }^{4}$ Department of Genetics and the Human Genetics Institute, Rutgers University Cell and DNA Repository, Rutgers University, New Jersey, USA. ${ }^{5}$ Departments of Genetics and Psychiatry, University of North Carolina, North Carolina, USA. ${ }^{6}$ Department of Pathology, VU University Medical Center, Amsterdam, The Netherlands.

Received: 12 March 2013 Accepted: 14 January 2014

Published: 17 January 2014

\section{References}

1. Choi BG, McLaughlin MA: Why men's hearts break: cardiovascular effects of sex steroids. Endocrinol Metab Clin North Am 2007, 36:365-377.

2. Whitacre CC: Sex differences in autoimmune disease. Nat Immunol 2001, 2:777-780.

3. Vink JM, Bartels M, van Beijsterveldt TCEM, van Dongen J, van Beek JHDA, Distel MA, de Moor MHM, Smit DJA, Minica CC, Ligthart L, Geels LM, Abdellaoui A, Middeldorp CM, Hottenga JJ, Willemsen G, de Geus EJC, Boomsma DI: Sex differences in genetic architecture of complex phenotypes? PLoS One 2012, 7:e47371.

4. Nolen-Hoeksema S: Emotion regulation and psychopathology: the role of gender. Annu Rev Clin Psychol 2012, 8:161-187.

5. Kim AM, Tingen CM, Woodruff TK: Sex bias in trials and treatment must end. Nature 2010, 465:688-689

6. Ober C, Loisel DA, Gilad Y: Sex-specific genetic architecture of human disease. Nat Rev Genet 2008, 9:911-922.

7. Carrel L, Willard HF: X-inactivation profile reveals extensive variability in X-linked gene expression in females. Nature 2005, 434:400-404.

8. Lahn BT, Page DC: Functional coherence of the human $Y$ chromosome. Science 1997, 278:675-680.

9. Jessen HM, Auger AP: Sex differences in epigenetic mechanisms may underlie risk and resilience for mental health disorders. Epigenetics 2011 6:857-861.

10. Ling G, Sugathan A, Mazor T, Fraenkel E, Waxman DJ: Unbiased, genome-wide In vivo mapping of transcriptional regulatory elements reveals sex differences in chromatin structure associated with sex-specific liver gene expression. Mol Cell Biol 2010, 30:5531-5544.

11. Sugathan A, Waxman DJ: Genome-wide analysis of chromatin states reveals distinct mechanisms of sex-dependent gene regulation in male and female mouse liver. Mol Cell Biol 2013, 33(18):3594-3610.

12. Ellegren $\mathrm{H}$, Parsch J: The evolution of sex-biased genes and sex-biased gene expression. Nat Rev Genet 2007, 8:689-698.

13. Gallach M, Domingues S, Betrán E: Gene duplication and the genome distribution of sex-biased genes. Int J Evol Biol 2011, 2011:989438.

14. Yang $X$, Schadt EE, Wang S, Wang H, Arnold AP, Ingram-Drake L, Drake TA, Lusis AJ: Tissue-specific expression and regulation of sexually dimorphic genes in mice. Genome Res 2006, 16:995-1004.

15. Mank JE, Hultin-Rosenberg L, Webster MT, Ellegren $\mathrm{H}$ : The unique genomic properties of sex-biased genes: insights from avian microarray data. BMC Genomics 2008, 9:148.

16. Parsch J, Ellegren $\mathrm{H}$ : The evolutionary causes and consequences of sex-biased gene expression. Nat Rev Genet 2013, 14:83-87.

17. Gatti DM, Zhao N, Chesler EJ, Bradford BU, Shabalin AA, Yordanova R, Lu L, Rusyn I: Sex-specific gene expression in the BXD mouse liver. Physiol Genomics 2010, 42:456-468.

18. Mozhui K, Lu L, Armstrong WE, Williams RW: Sex-specific modulation of gene expression networks in murine hypothalamus. Front Neurosci 2012, 6:63.

19. McRae AF, Matigian NA, Vadlamudi L, Mulley JC, Mowry B, Martin NG, Berkovic SF, Hayward NK, Visscher PM: Replicated effects of sex and genotype on gene expression in human lymphoblastoid cell lines. Hum Mol Genet 2007, 16:364-373.

20. Zhang W, Bleibel WK, Roe CA, Cox NJ, Dolan ME: Gender-specific differences in expression in human lymphoblastoid cell lines. Pharmacogenet Genomics 2007, 17:447-450.

21. Zhang Y, Klein K, Sugathan A, Nassery N, Dombkowski A, Zanger UM, Waxman DJ: Transcriptional profiling of human liver identifies sex-biased genes associated with polygenic dyslipidemia and coronary artery disease. PLoS One 2011, 6:e23506

22. Welle S, Tawil R, Thornton CA: Sex-related differences in gene expression in human skeletal muscle. PLOS One 2008, 3:e1385.

23. Gupta P, Harte AL, da Silva NF, Khan H, Barnett AH, Kumar S, Sturdee DW McTernan PG: Expression of calcitonin gene-related peptide, adrenomedullin, and receptor modifying proteins in human adipose tissue and alteration in their expression with menopause status. Menopause 2007, 14:1031-1038.

24. Dvornyk V, Liu Y, Lu Y, Shen H, Lappe JM, Lei S, Recker RR, Deng H: Effect of menopause on gene expression profiles of circulating monocytes: a pilot in vivo microarray study. J Genet Genomics 2007, 34:974-983.

25. Kósa JP, Balla B, Speer G, Kiss J, Borsy A, Podani J, Takács I, Lazáry A, Nagy Z, Bácsi $K$, Orosz L, Lakatos P: Effect of menopause on gene expression pattern in bone tissue of nonosteoporotic women. Menopause 2009, 16:367-377.

26. Dimas A, Nica A, Montgomery S, Stranger B, Raj T, Buil A, Giger T, Lappalainen T, Gutierrez-Arcelus M, McCarthy M, Dermitzakis E: Sex-biased genetic effects on gene regulation in humans. Genome Res 2012, 22(12):2368-2375.

27. Boomsma DI, Willemsen G, Sullivan PF, Heutink $P$, Meijer $P$, Sondervan D, Kluft C, Smit G, Nolen WA, Zitman FG, Smit JH, Hoogendijk WJ, van Dyck R, de Geus EJC, Penninx BWJH: Genome-wide association of major depression: description of samples for the GAIN major depressive disorder study: NTR and NESDA biobank projects. Eur J Hum Genet 2008, 16:335-342.

28. Langfelder P, Horvath S: WGCNA: an R package for weighted correlation network analysis. BMC Bioinformatics 2008, 9:559.

29. Nickel GC, Tefft D, Adams MD: Human PAML browser: a database of positive selection on human genes using phylogenetic methods. Nucleic Acids Res 2008, 36(Database issue):D800-D808.

30. Nielsen R, Bustamante C, Clark AG, Glanowski S, Sackton TB, Hubisz MJ, Fledel-Alon A, Tanenbaum DM, Civello D, White TJ, Sninsky J J, Adams MD, Cargill M: A scan for positively selected genes in the genomes of humans and chimpanzees. PLoS Biol 2005, 3:e170.

31. The Chimpanzee Sequencing and Analysis Consortium: Initial sequence of the chimpanzee genome and comparison with the human genome. Nature 2005, 437:69-87.

32. Lu T, Pan Y, Kao S-Y, Li C, Kohane I, Chan J, Yankner BA: Gene regulation and DNA damage in the ageing human brain. Nature 2004, 429:883-891.

33. Boraska V, Jerončić A, Colonna V, Southam L, Nyholt DR, Rayner NW, Perry JRB, Toniolo D, Albrecht E, Ang W, Bandinelli S, Barbalic M, Barroso I, Beckmann JS, Biffar R, Boomsma D, Campbell H, Corre T, Erdmann J, Esko T, Fischer K, Franceschini N, Frayling TM, Girotto G, Gonzalez JR, Harris TB, 
Heath AC, Heid IM, Hoffmann W, Hofman A, et al: Genome-wide metaanalysis of common variant differences between men and women. Hum Mol Genet 2012, 21:4805-4815.

34. Ranz JM, Castillo-Davis Cl, Meiklejohn CD, Hartl DL: Sex-dependent gene expression and evolution of the drosophila transcriptome. Science 2003, 300:1742-1745.

35. Zhang Y, Sturgill D, Parisi M, Kumar S, Oliver B: Constraint and turnover in sex-biased gene expression in the genus Drosophila. Nature 2007, 450:233-237.

36. Ramachandran S, Palanisamy V: Horizontal transfer of RNAs: exosomes as mediators of intercellular communication. Wiley Interdiscip Rev RNA 2012, 3:286-293.

37. Reinius B, Saetre P, Leonard JA, Blekhman R, Merino-Martinez R, Gilad Y, Jazin E: An evolutionarily conserved sexual signature in the primate brain. PLoS Genet 2008, 4:e1000100.

38. Morey C, Avner P: The demoiselle of X-inactivation: 50 years old and as trendy and mesmerising as ever. PLoS Genet 2011, 7:e1002212

39. Carroll JS, Meyer CA, Song J, Li W, Geistlinger TR, Eeckhoute J, Brodsky AS, Keeton EK, Fertuck KC, Hall GF, Wang Q, Bekiranov S, Sementchenko V, Fox EA, Silver PA, Gingeras TR, Liu XS, Brown M: Genome-wide analysis of estrogen receptor binding sites. Nat Genet 2006, 38:1289-1297.

40. Welboren W-J, van Driel MA, Janssen-Megens EM, van Heeringen SJ, Sweep FC, Span PN, Stunnenberg HG: ChIP-Seq of ERalpha and RNA polymerase II defines genes differentially responding to ligands. EMBO J 2009, 28:1418-1428.

41. Hah N, Danko CG, Core L, Waterfall JJ, Siepel A, Lis JT, Kraus WL: A rapid, extensive, and transient transcriptional response to estrogen signaling in breast cancer cells. Cell 2011, 145:622-634.

42. Anderson E: The role of oestrogen and progesterone receptors in human mammary development and tumorigenesis. Breast Cancer Res 2002, 4:197-201.

43. Prossnitz ER, Barton M: The G-protein-coupled estrogen receptor GPER in health and disease. Nat Rev Endocrinol 2011, 7:715-726.

44. Watanabe H, Takahashi E, Kobayashi M, Goto M, Krust A, Chambon P, Iquchi $\mathrm{T}$ : The estrogen-responsive adrenomedullin and receptor-modifying protein 3 gene identified by DNA microarray analysis are directly regulated by estrogen receptor. J Mol Endocrinol 2006, 36:81-89.

45. Schnoes KK, Jaffe IZ, Iyer L, Dabreo A, Aronovitz M, Newfell B, Hansen U, Rosano G, Mendelsohn ME: Rapid recruitment of temporally distinct vascular gene sets by estrogen. Mol Endocrinol 2008, 22:2544-2556.

46. Potocki M, Ziller R, Mueller C: Mid-regional pro-adrenomedullin in acute heart failure: a better biomarker or just another biomarker? Curr Heart Fail Rep 2012, 9:244-251.

47. Teng C: Lactoferrin: the path from protein to gene. Biometals 2010, 23:359-364.

48. Gibbons JA, Kanwar RK, Kanwar JR: Lactoferrin and cancer in different cancer models. Front Biosci (Schol Ed) 2011, 3:1080-1088.

49. Rodvold JJ, Mahadevan NR, Zanetti M: Lipocalin 2 in cancer: when good immunity goes bad. Cancer Lett 2012, 316:132-138.

50. Heslop CL, Frohlich JJ, Hill JS: Myeloperoxidase and C-reactive protein have combined utility for long-term prediction of cardiovascular mortality after coronary angiography. J Am Coll Cardiol 2010, 55:1102-1109.

51. Bonkhoff $H$, Berges $R$ : The evolving role of oestrogens and their receptors in the development and progression of prostate cancer. Eur Urol 2009, 55:533-542

52. Oklü R, Hesketh $R$ : The latent transforming growth factor beta binding protein (LTBP) family. Biochem J 2000, 352(Pt 3):601-610

53. Zhang Y, Laz EV, Waxman DJ: Dynamic, sex-differential STAT5 and BCL6 binding to sex-biased, growth hormone-regulated genes in adult mouse liver. Mol Cell Biol 2012, 32:880-896.

54. Meinhardt UJ, Ho KKY: Modulation of growth hormone action by sex steroids. Clin Endocrinol (Oxf) 2006, 65:413-422.

55. Leung K-C, Johannsson G, Leong GM, Ho KKY: Estrogen regulation of growth hormone action. Endocr Rev 2004, 25:693-721.

56. Ansar Ahmed S, Penhale WJ, Talal N: Sex hormones, immune responses, and autoimmune diseases. Mechanisms of sex hormone action. Am J Pathol 1985, 121:531-551.

57. Oertelt-Prigione $\mathrm{S}$ : The influence of sex and gender on the immune response. Autoimmun Rev 2012, 11:A479-A485.

58. Oliver JE, Silman AJ: Why are women predisposed to autoimmune rheumatic diseases? Arthritis Res Ther 2009, 11:252
59. Nishimoto N: Interleukin-6 in rheumatoid arthritis. Curr Opin Rheumatol 2006, 18:277-281.

60. Menke J, Kriegsmann J, Schimanski CC, Schwartz MM, Schwarting A, Kelley VR: Autocrine CSF-1 and CSF-1 receptor coexpression promotes renal cell carcinoma growth. Cancer Res 2012, 72:187-200.

61. Woldrich JM, Mallin K, Ritchey J, Carroll PR, Kane CJ: Sex differences in renal cell cancer presentation and survival: an analysis of the National Cancer Database, 1993-2004. J Urol 2008, 179:1709-1713. discussion 1713.

62. Brennan AJ, Chia J, Trapani JA, Voskoboinik I: Perforin deficiency and susceptibility to cancer. Cell Death Differ 2010, 17:607-615.

63. Kim T-D, Lee SU, Yun S, Sun H-N, Lee SH, Kim JW, Kim HM, Park S-K, Lee CW, Yoon SR, Greenberg PD, Choi I: Human microRNA-27a* targets Prf1 and GzmB expression to regulate NK-cell cytotoxicity. Blood 2011, 118:5476-5486.

64. Angiolillo DJ: The evolution of antiplatelet therapy in the treatment of acute coronary syndromes: from aspirin to the present day. Drugs 2012, 72:2087-2116.

65. Antithrombotic Trialists' Collaboration: Collaborative meta-analysis of randomised trials of antiplatelet therapy for prevention of death, myocardial infarction, and stroke in high risk patients. BMJ 2002, 324:71-86.

66. Stankovic S, Majkic-Singh N: Genetic aspects of ischemic stroke: coagulation, homocysteine, and lipoprotein metabolism as potential risk factors. Crit Rev Clin Lab Sci 2010, 47:72-123.

67. Penninx BWJH, Beekman ATF, Smit JH, Zitman FG, Nolen WA, Spinhoven $P$, Cuijpers P, De Jong PJ, Van Marwijk HWJ, Assendelft WJJ, Van Der Meer K, Verhaak P, Wensing M, De Graaf R, Hoogendijk WJ, Ormel J, Van Dyck R: The Netherlands Study of Depression and Anxiety (NESDA): rationale, objectives and methods. Int J Methods Psychiatr Res 2008, 17:121-140.

68. Boomsma DI, de Geus EJC, Vink JM, Stubbe JH, Distel MA, Hottenga J-J, Posthuma D, van Beijsterveldt TCEM, Hudziak JJ, Bartels M, Willemsen G: Netherlands twin register: from twins to twin families. Twin Res Hum Genet 2006, 9:849-857.

69. Willemsen G, de Geus EJC, Bartels M, van Beijsterveldt CEMT, Brooks Al, Estourgie-van Burk GF, Fugman DA, Hoekstra C, Hottenga J-J, Kluft K, Meijer P, Montgomery GW, Rizzu P, Sondervan D, Smit AB, Spijker S, Suchiman HED, Tischfield JA, Lehner T, Slagboom PE, Boomsma DI: The Netherlands Twin Register biobank: a resource for genetic epidemiological studies. Twin Res Hum Genet 2010, 13:231-245.

70. Spijker S, van de Leemput JCH, Hoekstra C, Boomsma DI, Smit AB: Profiling gene expression in whole blood samples following an in-vitro challenge. Twin Res 2004, 7:564-570.

71. Visscher PM, Benyamin B, White I: The use of linear mixed models to estimate variance components from data on twin pairs by maximum likelihood. Twin Res 2004, 7:670-674.

72. Shabalin AA: Matrix eQTL: ultra fast eQTL analysis via large matrix operations. Bioinformatics 2012, 28:1353-1358.

73. Abdellaoui A, Hottenga J-J, de Knijff P, Nivard MG, Xiao X, Scheet P, Brooks A, Ehli EA, Hu Y, Davies GE, Hudziak JJ, Sullivan PF, van Beijsterveldt T, Willemsen G, de Geus EJ, Penninx BWJH, Boomsma DI: Population structure, migration, and diversifying selection in the Netherlands. Eur J Hum Genet 2013, 21(11):1277-85.

74. Ashburner M, Ball CA, Blake JA, Botstein D, Butler H, Cherry JM, Davis AP, Dolinski K, Dwight SS, Eppig JT, Harris MA, Hill DP, Issel-Tarver L, Kasarskis A, Lewis S, Matese JC, Richardson JE, Ringwald M, Rubin GM, Sherlock G: Gene ontology: tool for the unification of biology. The gene ontology consortium. Nat Genet 2000, 25:25-29.

75. Maere S, Heymans K, Kuiper M: BiNGO: a Cytoscape plugin to assess overrepresentation of gene ontology categories in biological networks. Bioinformatics 2005, 21:3448-3449.

76. Miller JA, Cai C, Langfelder P, Geschwind DH, Kurian SM, Salomon DR, Horvath S: Strategies for aggregating gene expression data: the collapseRows R function. BMC Bioinformatics 2011, 12:322.

77. Zhang B, Horvath S: A general framework for weighted gene co-expression network analysis. Stat Appl Genet Mol Biol 2005, 4. Article17 Epub 2005 Aug 12.

doi:10.1186/1471-2164-15-33

Cite this article as: Jansen et al.: Sex differences in the human peripheral blood transcriptome. BMC Genomics 2014 15:33. 